Corollary 1. The absolute minimum value of $k$ is zero; this value is taken on if the midpoint of the line segments $\left(g_{t}, g_{t}^{\prime}\right)$ and $(g, 1 / \bar{g})$ coincide and is possible only for $T$ an elliptic transformation.

Proof. Substituting $m=-(\alpha \bar{\alpha}+\nu \bar{\nu}) /(2 \alpha \nu)$ into (2), we see that $k=0$ if $(a-\bar{a}) /(2 c)=-(\alpha \bar{\alpha}+\nu \bar{\nu}) /(2 \alpha \nu)$. Furthermore, we have $Q_{0}[-(\alpha \bar{\alpha}+\alpha \bar{\nu}) /(2 \alpha \nu)]>0$ for all $G$ and all $T$ of Fuchsian type, whereas $Q_{0}[(a-\bar{a}) /(2 c)]>0$ for $T$ elliptic only.

REMARK 3. Changing (2) to trigonometric form, one finds the discriminant of the resulting quadratic in $\rho$ to be

$$
f(k)=4\left(\alpha \nu e^{i \theta}+\bar{\alpha} \bar{\nu} e^{-i \theta}\right)^{2}-16 \alpha \bar{\alpha} \nu \bar{\nu}\left(1-k^{2}\right) .
$$

This is a perfect square if and only if $k=1$ or 0 ; hence (2) is factorable rationally in terms of the coefficients of $G$ in these two cases and only in them. The factors for $k=1$ are $Q_{5}$ and $Q_{6}$ of Theorem 1, and for $k=0$ they are immediate from (2).

State College of New Mexico

\title{
THE EQUATION $2^{x}-3^{y}=d^{*}$
}

\section{BY AARON HERSCHFELD}

1. Introduction. According to Dickson's History of the Theory of Numbers, $\dagger$ Leo Hebreus, or Levi Ben Gerson (1288-1344), proved that $3^{m} \pm 1 \neq 2^{n}$ if $m>2$, by showing that $3^{m} \pm 1$ has an odd prime factor. The problem had been proposed to him by Philipp von Vitry in the following form: All powers of 2 and 3 differ by more than unity except the pairs 1 and 2, 2 and 3, 3 and 4, 8 and 9. In 1923 an elegant short proof by Philip Franklin appeared in the American Mathematical Monthly.

In $1918 \mathrm{G}$. Polya $\S$ published a very general theorem which, as was later pointed out by S. Sivasankaranarayana Pillai,\| proved as special cases that the equations

* Presented to the Society, October 26, 1935.

† Vol. 2, p. 731; see J. Carlebach, Dissertation, Heidelberg, 1909, pp. 62-64.

‡ Vol. 30 (1923), p. 81, problem 2927.

§ Zur Arithmetische Untersuchung der Polynome, Mathematische Zeitschrift, vol. 1 (1918), pp. 143-148.

|| Journal of the Indian Mathematical Society, vol. 19 (1931), pp. 1-11. 


$$
\begin{aligned}
& a^{x}-b^{y}=d, \\
& 2^{x}-3^{y}=d,
\end{aligned}
$$

where $a$ and $b$ are fixed positive integers and $d \neq 0$, have at most a finite number of solutions in positive integers $(x, y)$. Moreover, Pillai gave an asymptotic formula for the finite number of solutions of the inequality $0<a^{x}-b^{y} \leqq n$, where $\log a / \log b$ is not rational.

In this paper we shall prove that if $|d|$ is sufficiently great, equation (2), for fixed $d$, can have at most one solution, while equation (1) can have no more than nine solutions.

The general existence theorems tell us in particular that the inequality $\left|2^{x}-3^{y}\right| \leqq n$ holds for only a finite number of pairs of positive integers $(x, y)$. But they do not tell us the precise values of such pairs $(x, y)$ nor exactly how many exist. We shall answer these questions for $n=10$ by solving the six equations $2^{x}-3^{y}=d, d= \pm 1, \pm 5, \pm 7$, since we may obviously exclude $d=0, \pm 2, \pm 3, \pm 4, \pm 6, \pm 8, \pm 9, \pm 10$. Similar methods apply to greater values, and we summarize some results for $n=100$.

2. Special Results. If $2^{x}-3^{y}=d$ and $x \geqq 3$, then $3^{y} \equiv-d$ $(\bmod 8)$. But $3^{n} \equiv 1$ or $3(\bmod 8)$. Hence $d \equiv 5$ or $d \equiv 7(\bmod 8)$. Consequently for $d=1,-5,-7$ there are no solutions of equation (1) such that $x \geqq 3$. We see therefore that these equations have only the solutions

$$
2^{2}-3=1, \quad 2^{2}-3^{2}=-5, \quad 2-3^{2}=-7 .
$$

There remain the cases $d=-1,5,7$. Suppose $2^{x}-3^{y}=-1$, so that $3^{y} \equiv 1\left(\bmod 2^{x}\right)$. But if $x>2,3$ belongs* to $2^{x-2}$ modulo $2^{x}$, that is, $2^{x-2}$ is the least integer $e$ such that $3^{e} \equiv 1$ modulo $2^{x}$. Therefore $y \geqq 2^{x-2}$, if $x>2$, and so

$$
3^{y}-1 \geqq 3^{2^{x-2}}-1 \geqq 3^{x}-1>2^{x},
$$

if $x \geqq 4$. Consequently $2^{x}-3^{y}=-1$ is impossible if $x \geqq 4$. Thus the only solutions are

$$
2-3=-1=2^{3}-3^{2} .
$$

Next consider the equation $2^{x}-3^{y}=5$. Obviously we have as two solutions $(x, y)=(3,1)$ and $(5,3)$. Suppose another solu-

* See Dickson, Introduction to the Theory of Numbers, pp. 16, 19. 
tion $(x, y)$ exists satisfying $2^{x}=3^{y}+5$ with $y>3$, and consequently $x>5$. Taking congruences modulo $2^{5}, 3^{y} \equiv-5\left(\bmod 2^{5}\right)$. But $3^{3}=2^{5}-5 \equiv-5\left(\bmod 2^{5}\right)$ and 3 belongs to 8 modulo $2^{5}$. Therefore $y=3+8 k$, where $k>0$. Now take congruences modulo $2^{6}$; since $x>5,3^{y} \equiv-5\left(\bmod 2^{6}\right)$. But $3^{3} \not \equiv-5\left(\bmod 2^{6}\right)$ and 3 belongs to $16\left(\bmod 2^{6}\right)$. Thus $k$ cannot be even and it follows that $y=11+16 k^{\prime}$.

Finally we take congruences modulo 17 , noting that 3 belongs to $16(\bmod 17)$, so that $2^{x}=3^{y}+5 \equiv-10+5 \equiv-5(\bmod$ 17). But we can easily verify that no power of 2 is congruent to $-5(\bmod 17)$. Hence we have a contradiction.

Lastly consider $2^{x}-3^{y}=7$, where $y>3$, so that $x>2$. Taking congruences modulo 3 and then modulo 4 , we see that both $x$ and $y$ are even. Therefore

$$
1 \leqq 2^{x / 2}-3^{y / 2}=7 /\left(2^{x / 2}+3^{y / 2}\right)<1,
$$

which is a contradiction. Hence the only solution is $2^{4}-3^{2}=7$.

We may summarize by tabulating our results:

$$
\begin{array}{l|l}
d & (x, y) \\
\hline-1 & (1,1) ;(3,2) \\
-5 & (2,2) \\
-7 & (1,2)
\end{array}
$$

\begin{tabular}{l|l}
$d$ & $(x, y)$ \\
\hline 1 & $(2,1)$ \\
5 & $(3,1) ;(5,3)$ \\
7 & $(4,2)$
\end{tabular}

3. General Theorems. We have now shown that when $x>5$, or $y>3$, then $\left|2^{x}-3^{y}\right|>10$. The author has verified in an unpublished paper that similar methods may be used to prove that if $x$ and $y$ are positive integers such that $x>8$ or $y>5$, then $\left|2^{x}-3^{y}\right|>100$. In no case were there found more than two solutions of the equation (2) for any fixed $d$ with $|d| \leqq 100$. We conclude by proving that if $|d|$ is sufficiently great, the equation $2^{x}-3^{y}=d$ cannot have more than one solution.

We shall use the property proved by S. S. Pillai in his 1931 paper, previously mentioned, that given any $\delta>0$ and integers $a$ and $b$ such that $\log a / \log b$ is not rational, there exists an integer $x_{1}=x_{1}(\delta)$ such that for all $x>x_{1}$ and all positive $\left(a^{x}-b^{y}\right)$,

$$
0<a^{x}-b^{y}>a^{x(1-\delta)} .
$$

Suppose that $2^{x}-3^{y}=2^{x}-3^{Y}=d, X>x, Y>y$. Then 


$$
\begin{array}{crl}
2^{X}-2^{x}=3^{Y}-3^{y}, & 2^{x}\left(2^{X-x}-1\right)=3^{y}\left(3^{Y-y}-1\right), \\
2^{X-x} \equiv 1\left(\bmod 3^{y}\right), & 3^{Y-y} \equiv 1\left(\bmod 2^{x}\right), \\
X-x \geqq 2 \cdot 3^{y-1}, \quad X \geqq 2 \cdot 3^{y-1}, & Y-y \geqq 2^{x-2}, \quad Y \geqq 2^{x-2},
\end{array}
$$

if $x>2$.

Consider first $d>0$. Choose any positive $\delta<1 / 2$ and let $x_{1}=x_{1}(\delta)$, where for all $x>x_{1}$ and $0<2^{x}-3^{y}$, we have $0<2^{x}-3^{y}>2^{x(1-\delta)}>2^{x / 2}$. Let us consider only the positive values of $d$ greater than $2^{x_{1}+5}$. Then

$$
\begin{aligned}
& 2^{x}>d>2^{x_{1}+5}, \quad x>x_{1}+5, \quad X>x>x_{1}, \\
& 2^{X}=3^{Y}+d>3^{Y} \geqq 3^{2^{x-2}}>2^{2^{x-2}}, \quad X>2^{x-2}>2 x,
\end{aligned}
$$

since $x>5$. Therefore $d=2^{X}-3^{Y}>2^{x / 2}>2^{x}>d$, a contradiction.

Next, for $d<0$, consider only values of $d$ such that $|d|>3^{y_{1}+2}$, where $y_{1}=y_{1}(\delta)$, so that for all $y>y_{1}$ and $0<3^{y}-2^{x}$, we have $0<3^{y}-2^{x}>3^{y(1-\delta)}>3^{y / 2}$. Hence, if (2) has two solutions,

$$
\begin{array}{cl}
3^{y}>|d|>3^{y_{1}+2}, \quad y>y_{1}+2, & Y>y>y_{1}, \\
3^{Y}=2^{X}-d>2^{X} \geqq 2^{2 \cdot 3^{y-1}}>3^{3^{y-1}}, &
\end{array}
$$

since $y>2$. Thus $-d=3^{Y}-2^{X}>3^{Y / 2}>3^{y}>-d$, a contradiction.

In conclusion we can say of the general equation $a^{x}-b^{y}=d$, that if $|d|$ is sufficiently great, this equation can have at most nine solutions. This is a simple consequence of the theorem due to C. L. Siegel, * that $a x^{n}-b y^{n}=k$ (fixed $n \geqq 3$ ) has at most one solution if $|a b|$ is sufficiently great. For if $|d|$ is large enough we can write equation (1) in the form of at least one of nine equations

$$
a^{i} A u^{3}-b^{j} B v^{3}=d, \quad(i, j=0,1,2),
$$

where $|A B|$ is so great that each of the nine equations must, by Siegel's theorem, have at most one solution.

Columbia University

* Abhandlungen Akademie Berlin, 1929, Nr. 1, 70 pp. 